\title{
ON GRAFT TRANSFORMATIONS DECREASING DISTANCE SPECTRAL RADIUS OF GRAPHS
}

\author{
YANNA WANG ${ }^{1}$ AND BO ZHOU ${ }^{2, *}$
}

\begin{abstract}
The distance spectral radius of a connected graph is the largest eigenvalue of its distance matrix. In this paper, we give several less restricted graft transformations that decrease the distance spectral radius, and determine the unique graph with minimum distance spectral radius among homeomorphically irreducible unicylic graphs on $n \geq 6$ vertices, and the unique tree with minimum distance spectral radius among trees on $n$ vertices with given number of vertices of degree two, respectively.
\end{abstract}

Mathematics Subject Classification. 05C50, 15A48.

Received November 21, 2020. Accepted May 27, 2021.

\section{INTRODUCTION}

We consider simple, finite, undirected and connected graphs. For $u \in V(G)$, let $N_{G}(u)$ be the set of neighbors of $u$ in $G$. The degree of a vertex $u$ in $G$, denoted by $\operatorname{deg}_{G}(u)$, is the number of edges incident to $u$ in $G$.

A homeomorphically irreducible tree is a tree with no vertex of degree two [6]. A homeomorphically irreducible unicylic graph is a unicylic graph with no vertex of degree two.

Let $G$ be a connected graph on $n$ vertices. For $u, v \in V(G)$, the distance between $u$ and $v$ in $G$, denoted by $d_{G}(u, v)$, is the length of a shortest path connecting them in $G$. In particular, $d_{G}(u, u)=0$. The distance spectrum of $G$ is the spectrum of the distance matrix of $G$, defined as the $n$ by $n$ symmetric matrix $D(G)=$ $\left(d_{G}(u, v)\right)_{u, v \in V(G)}$. The distance spectral radius of $G$, denoted by $\rho(G)$, is the largest distance eigenvalue of $G$. Graham and Pollack [5] studied the distance spectrum for the first time due to its connection to a data communication problem. Now the distance spectrum has been studied extensively, see the survey [1]. Particularly, the distance spectral radius has received much attention. Graphs with minimum and/or maximum distance spectral radius have been determined for some classes of graphs, see, e.g., [2,7,11-15]. Yu et al. [16] determined the graphs with minimum and maximum distance spectral radius among unicylic graphs. Lin and Zhou [8] determined the trees with maximum distance spectral radius among trees on $n$ vertices with given number of vertices of degree two.

To determine the structure of the graphs with minimum or maximum distance spectral radius in some class of graphs, we usually suppose the graph does not have the structure and perform surgery to obtain a graph for

Keywords. Distance spectral radius, distance matrix, graft transformation, homeomorphically irreducible unicylic graph.

1 Basic Courses Department, Guangdong Communication Polytechnic, Guangzhou 510650, P.R. China.

2 School of Mathematical Sciences, South China Normal University, Guangzhou 510631, P.R. China.

*Corresponding author: zhoubo@scnu.edu.cn 
which the distance spectral radius is decreased or increased. Such surgery is known as graft transformation(s) in the literature, see, e.g., $[3,4,9,14,16]$.

In this paper, we propose some graft transformations in a more elaborate way. We propose some graft transformations with less restricted conditions that decrease the distance spectral radius, and as applications, we identify the unique graphs that minimize the distance spectral radius among homeomorphically irreducible unicylic graphs on $n \geq 6$ vertices, and among trees on $n$ vertices with given number of vertices of degree two, respectively.

\section{Preliminaries}

Let $G$ be a connected graph with $V(G)=\left\{v_{1}, \ldots, v_{n}\right\}$. Since $D(G)$ is irreducible, by Perron-Frobenius theorem, $\rho(G)$ is simple and there is a unique unit positive eigenvector corresponding to $\rho(G)$, which is called the distance Perron vector of $G$, denoted by $x(G)$. If $y=\left(y_{v_{1}}, \ldots, y_{v_{n}}\right)^{\top} \in \mathbb{R}^{n}$ is unit and has at least one nonnegative entry, then by Rayleigh's principle, we have $\rho(G) \geq y^{T} D(G) y$ with equality if and only if $y=x(G)$. If $x=x(G)$, then for each $u \in V(G)$, we have $\rho(G) x_{u}=\sum_{v \in V(G)} d_{G}(u, v) x_{v}$, which is called the distance eigenequation of $G$ at $u$.

Let $N \subseteq\left(N_{G}(u) \backslash N_{G}(v)\right) \backslash\{v\}$. Let $G^{\prime}=G-u w+v w$ for $w \in N$. We say that $G^{\prime}$ is obtained from $G$ by moving edge $u w$ at $u$ from $u$ to $v$.

For a connected graph $G$ with $V_{1} \subseteq V(G)$, let $\sigma_{G}\left(V_{1}\right)$ be the sum of the entries of the distance Perron vector of $G$ corresponding to the vertices in $V_{1}$. Furthermore, if all the vertices of $V_{1}$ induce a connected subgraph $H$ of $G$, then we write $\sigma_{G}(H)$ instead of $\sigma_{G}\left(V_{1}\right)$.

A component of a graph is a maximal connected subgraph, and a cut edge is an edge of a graph whose removal increases the number of components of the graph.

For a connected graph $G$, let $s(G)$ be the minimum row sum of $D(G)$. In [17], $\frac{s(G)}{n}$ is called the mean vertex deviation of $G$, where $n=|V(G)|$. It is known that $\rho(G) \geq s(G)$, see Theorem 1.1 in page 24 of [10].

\section{Graft transformations that DECREASE the Distance SPECTRAL RADiUs}

Firstly, we give a result related to (the entries of) the distance Perron vector, which will be frequently used in the subsequent proofs.

Lemma 3.1. Suppose that $v, w$ be two non-adjacent neighbors of vertex $u$ in a connected graph $G$. Let $x=x(G)$. Then $x_{w}+x_{u}-x_{v}>0$.

Proof. Let $V_{1}=V(G) \backslash\{u, v, w\}$. For $z \in V_{1}$, one has $d_{G}(w, z) \geq 1$ and $d_{G}(u, z)-d_{G}(v, z) \geq-d_{G}(u, v)=-1$, so $d_{G}(w, z)+d_{G}(u, z)-d_{G}(v, z) \geq 0$. From the distance eigenequations of $G$ at $w, u$ and $v$, we have

$$
\begin{aligned}
& \rho(G) x_{w}=x_{u}+2 x_{v}+\sum_{z \in V_{1}} d_{G}(w, z) x_{z}, \\
& \rho(G) x_{u}=x_{w}+x_{v}+\sum_{z \in V_{1}} d_{G}(u, z) x_{z}, \\
& \rho(G) x_{v}=2 x_{w}+x_{u}+\sum_{z \in V_{1}} d_{G}(v, z) x_{z} .
\end{aligned}
$$

Thus

$$
\begin{aligned}
\rho(G)\left(x_{w}+x_{u}-x_{v}\right) & =-x_{w}+3 x_{v}+\sum_{z \in V_{1}}\left(d_{G}(w, z)+d_{G}(u, z)-d_{G}(v, z)\right) x_{z} \\
& \geq-x_{w}+3 x_{v},
\end{aligned}
$$

which implies $(\rho(G)+1)\left(x_{w}+x_{u}-x_{v}\right) \geq x_{u}+2 x_{v}>0$. So it follows that $x_{w}+x_{u}-x_{v}>0$. 
Now we turn our attention to some graft transformations that decrease the distance spectral radius.

Theorem 3.2. Let $G$ be a graph consisting of nontrivial connected graphs $G_{1}$ and $G_{2}$ sharing a unique vertex $u$ such that $E(G)=E\left(G_{1}\right) \cup E\left(G_{2}\right)$. Suppose that u has neighbor $v$ of degree at least two in $G_{2}$ satisfying that, for any $z \in V\left(G_{2}\right) \backslash\{u, v\}, d_{G}(u, z) \neq d_{G}(v, z)$. Let $G^{\prime}$ be the graph obtained from $G$ by moving all the edges at $v$ except uv from $v$ to $u$. Then $\rho(G)>\rho\left(G^{\prime}\right)$.

Proof. Let $w$ be a neighbor of $u$ in $G_{1}$. Let $x=x\left(G^{\prime}\right)$. By Lemma 3.1, we have $x_{w}+x_{u}-x_{v}>0$.

Let $S=\left\{z \in V\left(G_{2}\right) \backslash\{u, v\}: d_{G}(u, z)-d_{G}(v, z)=1\right\}$. Let $z$ be a neighbor of $v$ in $G_{2}$ different from $u$. As $d_{G}(u, z) \neq d_{G}(v, z)=1$ and $d_{G}(u, z) \leq d_{G}(v, z)+d_{G}(u, v)=2$, one has $d_{G}(u, z)=2$. So $z \in S$ and $S \neq \emptyset$.

Claim. $\frac{1}{2} x^{\top}\left(D(G)-D\left(G^{\prime}\right)\right) x \geq \sigma_{G^{\prime}}(S)\left(\sigma_{G^{\prime}}\left(G_{1}\right)-x_{v}\right)$.

Note first that, as we pass from $G$ to $G^{\prime}$, the distance between a vertex of $S$ and a vertex of $V\left(G_{1}\right)$ is decreased by 1 , and the distance between a vertex of $S$ and $v$ is increased by 1 . So, to prove the claim, we need only to show that the distance between any other vertex pairs is decreased or remains unchanged.

It is evident that the distance between any two vertices in $V\left(G_{1}\right) \cup\{v\}$ remains unchanged as we pass from $G$ to $G^{\prime}$.

Suppose that $z_{1}, z_{2} \in V\left(G_{2}\right) \backslash\{u, v\}$. Let $P$ be a path from $z_{1}$ to $z_{2}$ with length $d_{G}\left(z_{1}, z_{2}\right)$ in $G$. If $v$ lies outside $P$, then $P$ is also a path connecting $z_{1}$ and $z_{2}$ in $G^{\prime}$. Suppose that $v$ lies on $P$. If $u$ lies outside $P$, then the path obtained from $P$ by replacing $v$ with $u$ is a path connecting $z_{1}$ and $z_{2}$ in $G^{\prime}$. Otherwise, $u$ lies on $P$. In this case, $u v$ appears to be an edge on $P$. So the path obtained from $P$ by deleting $v$ is a path connecting $z_{1}$ and $z_{2}$ in $G^{\prime}$. So the distance between any two vertices in $V\left(G_{2}\right) \backslash\{u, v\}$ is decreased or remains unchanged as we pass from $G$ to $G^{\prime}$.

Suppose that $z \in \bar{S}:=\left(V\left(G_{2}\right) \backslash\{u, v\}\right) \backslash S$ (if $\left.\bar{S} \neq \emptyset\right)$. Then $d_{G}(u, z)-d_{G}(v, z) \neq 0,1$. As $\left|d_{G}(u, z)-d_{G}(v, z)\right| \leq$ $d_{G}(u, v)=1$, one has $d_{G}(u, z)-d_{G}(v, z)=-1$. Let $P$ be a path from $u$ to $z$ with length $d_{G}(u, z)$ in $G$. Then $v$ lies outside $P$, so $P$ is also a path from $u$ to $z$ in $G^{\prime}$. Therefore, the distance between a vertex in $\bar{S}$ and a vertex in $V\left(G_{1}\right) \cup\{v\}$ is decreased or remains unchanged as we pass from $G$ to $G^{\prime}$.

Now we complete the proof of the claim. As $\rho(G) \geq x^{\top} D(G) x$ and $\rho\left(G^{\prime}\right)=x^{\top} D\left(G^{\prime}\right) x$, one has $\rho(G)-\rho\left(G^{\prime}\right) \geq$ $x^{\top}\left(D(G)-D\left(G^{\prime}\right)\right) x$. So, by the claim,

$$
\begin{aligned}
\frac{1}{2}\left(\rho(G)-\rho\left(G^{\prime}\right)\right) & \geq \frac{1}{2} x^{\top}\left(D(G)-D\left(G^{\prime}\right)\right) x \\
& \geq \sigma_{G^{\prime}}(S)\left(\sigma_{G^{\prime}}\left(G_{1}\right)-x_{v}\right) \\
& \geq \sigma_{G^{\prime}}(S)\left(x_{w}+x_{u}-x_{v}\right) \\
& >0
\end{aligned}
$$

and thus $\rho(G)>\rho\left(G^{\prime}\right)$.

In the following, we give some consequences of Theorem 3.2.

Corollary 3.3 ([14]). Let $G$ be a connected graph with a cut edge uv that is not a pendant edge. Let $G^{\prime}$ be the graph obtained from $G$ by moving all edges at $v$ except uv from $v$ to $u$. Then $\rho(G)>\rho\left(G^{\prime}\right)$.

Proof. Let $G_{1}$ be the component of $G-u v$ containing $u$ and $G_{2}$ be the subgraph of $G$ induced by $V(G) \backslash\left(V\left(G_{1}\right) \backslash\right.$ $\{u\})$. Note that $d_{G}(u, z)=d_{G}(v, z)+1$ for any $z \in V\left(G_{2}\right) \backslash\{u, v\}$. So the result follows from Theorem 3.2.

A chain in a graph $G$ is a cycle $C$ such that $G-E(C)$ has exactly $|V(C)|$ components. Length of the cycle $C$ is the length of the chain. The following is Lemma 3.3 of [3].

Corollary 3.4. Let $G$ be a connected graph with a chain $C$ of even length. Let uv be an edge on the chain $C$. Suppose that $\operatorname{deg}_{G}(u) \geq 3$. If $G^{\prime}=G-\left\{v w: w \in N_{G}(v) \backslash\{u\}\right\}+\left\{u w: w \in N_{G}(v) \backslash\{u\}\right\}$, then $\rho(G)>\rho\left(G^{\prime}\right)$. 
Proof. Let $G_{1}$ be the component of $G-E(C)$ containing $u$, and $G_{2}$ be the subgraph of $G$ induced by $V(G) \backslash$ $\left(V\left(G_{1}\right) \backslash\{u\}\right)$. Let $z \in V\left(G_{2}\right) \backslash\{u, v\}$. Note that $d_{G}(u, z)-d_{G}(v, z)=d_{G}(u, w)-d_{G}(v, w)$ for some $w$ on $C$. If $z$ lies on $C$, this is evident as $w=z$, otherwise, $w$ is the vertex on $C$ such that its distance to $z$ is minimum among all the distances between vertices of $C$ and $z$ in $G$. As $C$ is a chain, the shortest path connecting $u$ ( $v$, respectively) and $w$ contains only vertices on $C$. As the length of $C$ is even, there is a shortest path connecting $u$ and $w$ passing through $v$ or a shortest path connecting $v$ and $w$ passing through $u$, implying that $\left|d_{G}(u, w)-d_{G}(v, w)\right|=1$, so $d_{G}(u, z) \neq d_{G}(v, z)$. So the result follows from Theorem 3.2.

Corollary 3.5. Let $H$ be a graph consisting of two nontrivial connected graphs $H_{1}$ and $H_{2}$ sharing a unique vertex $u$ such that $E(H)=E\left(H_{1}\right) \cup E\left(H_{2}\right)$. Suppose that $u v_{1}, \ldots$, uv $v_{k}$ are pendant edges in $H_{1}$, where $k \geq 1$ and that $N_{H_{2}}(u)=N_{1} \cup N_{2}$, where $N_{1}, N_{2} \neq \emptyset$ and $N_{1} \cap N_{2}=\emptyset$. Let

$$
G=H-\left\{u w: w \in N_{1}\right\}+\left\{v_{k} w: w \in N_{1}\right\}
$$

or

$$
G=H-\left\{u w: w \in N_{2}\right\}+\left\{v_{k} w: w \in N_{2}\right\} .
$$

For any vertex $w \in V\left(H_{2}\right) \backslash\{u\}$, if all the paths from $u$ to $w$ with the length $d_{H}(u, w)$ pass only through vertices in $N_{1}$ or pass only through vertices in $N_{2}$, then $\rho(G)>\rho(H)$.

Proof. Assume that $G=H-\left\{u w: w \in N_{2}\right\}+\left\{v_{k} w: w \in N_{2}\right\}$. Then $H$ is obtainable from $G$ by moving all the edges at $v_{k}$ except $u v_{k}$ from $v_{k}$ to $u$. Let $G_{1}=H_{1}-v_{k}$ and let $G_{2}$ be the subgraph of $G$ induced by $V\left(H_{2}\right) \cup\left\{v_{k}\right\}$. Suppose that $z \in V\left(G_{2}\right) \backslash\left\{u, v_{k}\right\}$, i.e., $z \in V\left(H_{2}\right) \backslash\{u\}$. Note that any shortest path from $u$ to $z$ in $H_{2}$ goes through only vertices in $N_{1}$ or $N_{2}$. Correspondingly, any shortest path from $u$ to $z$ in $G_{2}$ goes through only vertices in $N_{1}$, so $d_{G}(u, z)=d_{G}\left(v_{k}, z\right)-1<d_{G}\left(v_{k}, z\right)$, or any shortest path from $u$ to $z$ in $G_{2}$ goes through only vertices in $N_{2}$, so $d_{G}(u, z)=d_{G}\left(v_{k}, z\right)+1>d_{G}\left(v_{k}, z\right)$. Now by Theorem 3.2, $\rho(G)>\rho(H)$.

If $k \geq 2$, then Corollary 3.5 becomes Theorem 2.4 of [16].

Corollary 3.3 may be generalized as the following version.

Theorem 3.6. Let $G$ be the graph obtained from vertex disjoint nontrivial connected graphs $G_{1}$ and $G_{2}$ with $u \in V\left(G_{1}\right)$ and $v \in V\left(G_{2}\right)$ by adding a path $P_{t}=v_{1} \ldots v_{t}$ with $v_{1}=u$ and $v_{t}=v$, where $t \geq 2, V\left(G_{1}\right) \cap V\left(P_{t}\right)=$ $\left\{v_{1}\right\}$ and $V\left(G_{2}\right) \cap V\left(P_{t}\right)=\left\{v_{t}\right\}$. Let $G^{\prime}$ be the graph obtained from $G$ by moving all the edges at $v_{t}$ in $E\left(G_{2}\right)$ from $v_{t}$ to $v_{1}$. Then $\rho(G)>\rho\left(G^{\prime}\right)$.

Proof. Let $x=x\left(G^{\prime}\right)$. Let $p=\left\lfloor\frac{t}{2}\right\rfloor$ and $p_{1}=\left\lceil\frac{t}{2}\right\rceil$.

Let $\Gamma=\sum_{i=1}^{p} x_{v_{i}}+\sigma_{G^{\prime}}\left(V\left(G_{1}\right) \backslash\left\{v_{1}\right\}\right)+\sigma_{G^{\prime}}\left(V\left(G_{2}\right) \backslash\left\{v_{t}\right\}\right)-\sum_{i=p_{1}+1}^{t} x_{v_{i}}$.

From the distance eigenequations of $G^{\prime}$ at $v_{p_{1}+1}$ and $v_{p}$, we have

$$
\rho\left(G^{\prime}\right)\left(x_{v_{p_{1}+1}}-x_{v_{p}}\right)=\left(p_{1}+1-p\right) \Gamma .
$$

For $i=1, \ldots, p-1$, from the distance eigenequations of $G^{\prime}$ at $v_{t+1-i}, v_{i}, v_{t+1-(i+1)}$ and $v_{i+1}$, we have

$$
\begin{aligned}
\rho\left(G^{\prime}\right) & \left(\left(x_{v_{t+1-i}}-x_{v_{i}}\right)-\left(x_{v_{t+1-(i+1)}}-x_{v_{i+1}}\right)\right) \\
& =\rho\left(G^{\prime}\right)\left(x_{v_{t+1-i}}-x_{v_{i}}\right)-\rho\left(G^{\prime}\right)\left(x_{v_{t+1-(i+1)}}-x_{v_{i+1}}\right) \\
& =2\left(\sum_{j=1}^{i} x_{v_{j}}+\sigma_{G^{\prime}}\left(V\left(G_{1}\right) \backslash\left\{v_{1}\right\}\right)+\sigma_{G^{\prime}}\left(V\left(G_{2}\right) \backslash\left\{v_{t}\right\}\right)-\sum_{j=t+1-i}^{t} x_{v_{j}}\right) \\
& =2 \Gamma-2 \sum_{j=i+1}^{p} x_{v_{j}}+2 \sum_{j=p_{1}+1}^{t-i} x_{v_{j}}
\end{aligned}
$$




$$
=2 \Gamma+2 \sum_{j=i+1}^{p}\left(x_{v_{t+1-j}}-x_{v_{j}}\right) .
$$

We claim that $x_{v_{t+1-i}}-x_{v_{i}}$ and $\Gamma$ have common sign for $i=1, \ldots, p$ by induction on $i$. If $i=p$, then it follows from (3.1). Suppose that $1 \leq i \leq p-1$, and $x_{v_{t+1-j}}-x_{v_{j}}$ and $\Gamma$ have common sign for $i+1 \leq j \leq p$. So $\sum_{j=i+1}^{p}\left(x_{v_{t+1-j}}-x_{v_{j}}\right)$ and $\Gamma$ have common sign. Thus, from (3.2), $\left(x_{v_{t+1-i}}-x_{v_{i}}\right)-\left(x_{v_{t+1-(i+1)}}-x_{v_{i+1}}\right)$ and $\Gamma$ have common sign. This, together with the induction assumption that $x_{v_{t+1-(i+1)}}-x_{v_{i+1}}$ and $\Gamma$ have common sign, implies that $x_{v_{t+1-i}}-x_{v_{i}}$ and $\Gamma$ have common sign.

Note that

$$
\Gamma>\sum_{i=1}^{p} x_{v_{i}}-\sum_{i=p_{1}+1}^{t} x_{v_{i}}=-\sum_{i=1}^{p}\left(x_{v_{t+1-i}}-x_{v_{i}}\right) .
$$

This requires the above common sign to be + . Again, from (3.1) and (3.2), we have $x_{v_{t+1-i}}-x_{v_{i}}>x_{v_{t+1-(i+1)}}-$ $x_{v_{i+1}}>0$ for $i=1, \ldots, p-1$, i.e., $0<x_{v_{t+1-i}}-x_{v_{i}}<x_{v_{t+2-i}}-x_{v_{i-1}}$ for $i=2, \ldots, p$. It follows that for $i=2, \ldots, p$,

$$
0<x_{v_{t+1-i}}-x_{v_{i}}<x_{v_{t}}-x_{v_{1}} .
$$

As $P_{t}=v_{1} \ldots v_{t}$ is a proper induced subgraph of $G^{\prime}$ and $d_{P_{t}}\left(v_{i}, v_{j}\right)=d_{G^{\prime}}\left(v_{i}, v_{j}\right)$ for any $1 \leq i<j \leq t$, we have $s\left(G^{\prime}\right)>s\left(P_{t}\right)$. Note that $\rho\left(G^{\prime}\right) \geq s\left(G^{\prime}\right)\left([10]\right.$, Thm. 1.1 in p. 24) and $s\left(P_{t}\right)=\left\lfloor\frac{t^{2}}{4}\right\rfloor[17]$. So

$$
\rho\left(G^{\prime}\right) \geq s\left(G^{\prime}\right)>s\left(P_{t}\right)=\left\lfloor\frac{t^{2}}{4}\right\rfloor .
$$

As we pass from $G$ to $G^{\prime}$, the distance between a vertex of $V\left(G_{2}\right) \backslash\left\{v_{t}\right\}$ and a vertex of $V\left(G_{1}\right) \backslash\left\{v_{1}\right\}$ is decreased by $t-1$, the distance between a vertex of $V\left(G_{2}\right) \backslash\left\{v_{t}\right\}$ and $v_{i}$ for $i=1, \ldots, t$ is decreased by $t-2 i+1$, and the distances between all other vertex pairs remain unchanged. Let $A=(t-1) \sigma_{G^{\prime}}\left(V\left(G_{1}\right) \backslash\left\{v_{1}\right\}\right)+\sum_{i=1}^{p}(t-$ $2 i+1)\left(x_{v_{i}}-x_{v_{t+1-i}}\right)$, i.e.,

$$
A=(t-1) \sigma_{G^{\prime}}\left(V\left(G_{1}\right) \backslash\left\{v_{1}\right\}\right)+\sum_{i=1}^{t}(t-2 i+1) x_{v_{i}} .
$$

So

$$
\begin{aligned}
\frac{1}{2}\left(\rho(G)-\rho\left(G^{\prime}\right)\right) & \geq \frac{1}{2} x^{\top}\left(D(G)-D\left(G^{\prime}\right)\right) x \\
& =\sigma_{G^{\prime}}\left(V\left(G_{2}\right) \backslash\left\{v_{t}\right\}\right)\left((t-1) \sigma_{G^{\prime}}\left(V\left(G_{1}\right) \backslash\left\{v_{1}\right\}\right)+\sum_{i=1}^{t}(t-2 i+1) x_{v_{i}}\right) \\
& =\sigma_{G^{\prime}}\left(V\left(G_{2}\right) \backslash\left\{v_{t}\right\}\right) \cdot A .
\end{aligned}
$$

Let $G^{*}$ be the graph obtained from $G$ by moving all the edges at $v_{1}$ in $E\left(G_{1}\right)$ from $v_{1}$ to $v_{t}$. Let $y=x\left(G^{*}\right)$. Let $B=(t-1) \sigma_{G^{*}}\left(V\left(G_{2}\right) \backslash\left\{v_{t}\right\}\right)+\sum_{i=1}^{p}(t-2 i+1)\left(y_{v_{t+1-i}}-y_{v_{i}}\right)$, i.e.,

$$
B=(t-1) \sigma_{G^{*}}\left(V\left(G_{2}\right) \backslash\left\{v_{t}\right\}\right)-\sum_{i=1}^{t}(t-2 i+1) y_{v_{i}} .
$$

By the similar arguments as above, we have

$$
\frac{1}{2}\left(\rho(G)-\rho\left(G^{*}\right)\right) \geq \frac{1}{2} y^{\top}\left(D(G)-D\left(G^{*}\right)\right) y
$$




$$
\begin{aligned}
& =\sigma_{G^{*}}\left(V\left(G_{1}\right) \backslash\left\{v_{1}\right\}\right)\left((t-1) \sigma_{G^{*}}\left(V\left(G_{2}\right) \backslash\left\{v_{t}\right\}\right)-\sum_{i=1}^{t}(t-2 i+1) y_{v_{i}}\right) \\
& =\sigma_{G^{\prime}}\left(V\left(G_{1}\right) \backslash\left\{v_{1}\right\}\right) \cdot B .
\end{aligned}
$$

It is evident that $\phi: V\left(G^{\prime}\right) \rightarrow V\left(G^{*}\right)$ defined by

$$
\phi(w)=\left\{\begin{array}{lll}
w & \text { if } \quad w \in V\left(G_{1}\right) \backslash\{u\} \text { or } w \in V\left(G_{2}\right) \backslash\{v\}, \\
v_{t+1-i} & \text { if } \quad w=v_{i} \text { with } i=1, \ldots, t
\end{array}\right.
$$

is an isomorphism from $G^{\prime}$ to $G^{*}$. Let $M$ be the permutation matrix associated to this isomorphism. That is, the nonzero entries of $M$ are $M_{w w}=1$ if $w \in V\left(G_{1}\right) \backslash\{u\}$ or $w \in V\left(G_{2}\right) \backslash\{v\}, M_{v_{i} v_{t+1-i}}=1$ for $i=1, \ldots, t$. Then $M^{T} D\left(G^{\prime}\right) M=D\left(G^{*}\right)$. As $\rho\left(G^{\prime}\right)=x^{\top} D\left(G^{\prime}\right) x=(M x)^{\top} D\left(G^{*}\right) M x, M x$ is the distance Perron vector of $G^{*}$ and so $y=M x$ by the Perron-Frobenius theorem. That is, $y_{w}=x_{w}$ if $w \in V\left(G_{1}\right) \backslash\{u\}$ or $w \in V\left(G_{2}\right) \backslash\{v\}$, and $y_{v_{i}}=x_{v_{t+1-i}}$. Then $B=(t-1) \sigma_{G^{\prime}}\left(V\left(G_{2}\right) \backslash\left\{v_{t}\right\}\right)+\sum_{i=1}^{p}(t-2 i+1)\left(x_{v_{i}}-x_{v_{t+1-i}}\right)$. From the distance eigenequations of $G^{\prime}$ at $v_{t}$ and $v_{1}$, we have

$$
\begin{aligned}
\rho\left(G^{\prime}\right)\left(x_{v_{t}}-x_{v_{1}}\right)= & (t-1)\left(\sigma_{G^{\prime}}\left(V\left(G_{1}\right) \backslash\left\{v_{1}\right\}\right)+\sigma_{G^{\prime}}\left(V\left(G_{2}\right) \backslash\left\{v_{t}\right\}\right)\right) \\
& +\sum_{i=1}^{p}(t-2 i+1)\left(x_{v_{i}}-x_{v_{t+1-i}}\right) \\
= & A+B-\sum_{i=1}^{p}(t-2 i+1)\left(x_{v_{i}}-x_{v_{t+1-i}}\right) \\
= & A+B+\sum_{i=1}^{p}(t-2 i+1)\left(x_{v_{t+1-i}}-x_{v_{i}}\right) .
\end{aligned}
$$

Then, by (3.3) and (3.4), we have

$$
\begin{aligned}
A+B & =\rho\left(G^{\prime}\right)\left(x_{v_{t}}-x_{v_{1}}\right)-\sum_{i=1}^{p}(t-2 i+1)\left(x_{v_{t+1-i}}-x_{v_{i}}\right) \\
& \geq \rho\left(G^{\prime}\right)\left(x_{v_{t}}-x_{v_{1}}\right)-\sum_{i=1}^{p}(t-2 i+1)\left(x_{v_{t}}-x_{v_{1}}\right) \\
& =\left(\rho\left(G^{\prime}\right)-\sum_{i=1}^{p}(t-2 i+1)\right)\left(x_{v_{t}}-x_{v_{1}}\right) \\
& =\left(\rho\left(G^{\prime}\right)-\left\lfloor\frac{t^{2}}{4}\right\rfloor\right)\left(x_{v_{t}}-x_{v_{1}}\right) \\
& >0 .
\end{aligned}
$$

Thus $A>0$ or $B>0$. Now the result follows from (3.5) and (3.6).

Now we present the third graft transformation and consider its effect on the distance spectral radius.

Theorem 3.7. Let $G$ be a graph consisting of two nontrivial connected graphs $G_{1}$ and $G_{2}$ sharing a unique vertex $u$ such that $E(G)=E\left(G_{1}\right) \cup E\left(G_{2}\right)$. Suppose that $N_{G_{2}}(u)=\left\{v_{1}, v_{2}\right\}, \operatorname{deg}_{G_{2}}\left(v_{i}\right) \geq 2$ for $i=1,2$, $v_{1}$ and $v_{2}$ are not adjacent, and for any $w \in V\left(G_{2}\right) \backslash\{u\}, d_{G_{2}}\left(v_{1}, w\right) \neq d_{G_{2}}\left(v_{2}, w\right)$. Let $G^{\prime}$ be the graph obtained from $G$ by moving all the edges at $v_{i}$ except $u v_{i}$ from $v_{i}$ to $u$ for each $i=1,2$. Then $\rho(G)>\rho\left(G^{\prime}\right)$. 
Proof. For $i=1,2$, let $S_{i}=\left\{z \in V\left(G_{2}\right) \backslash\left\{v_{i}\right\}: d_{G_{2}}(u, z)-d_{G_{2}}\left(v_{i}, z\right)=1\right\}$. As $\operatorname{deg}_{G_{2}}\left(v_{i}\right) \geq 2$, one has $S_{i} \neq \emptyset$ for $i=1,2$. As $d_{G_{2}}\left(v_{1}, w\right) \neq d_{G_{2}}\left(v_{2}, w\right)$ for any $w \in V\left(G_{2}\right) \backslash\{u\}$, one has $S_{1} \cap S_{2}=\emptyset$.

Choose $w \in N_{G_{1}}(u)$. Let $x=x\left(G^{\prime}\right)$, then by Lemma 3.1, we have $x_{w}+x_{u}-x_{v_{i}}>0$ for $i=1,2$.

As we pass from $G$ to $G^{\prime}$, for $i=1,2$, the distance between a vertex of $S_{i}$ and a vertex of $V\left(G_{1}\right)$ is decreased by 1 , the distance between a vertex of $S_{i}$ and $v_{i}$ is increased by 1 , and the distance between any other vertex pair is decreased or remains unchanged. So

$$
\begin{aligned}
\frac{1}{2}\left(\rho(G)-\rho\left(G^{\prime}\right)\right) & \geq \frac{1}{2} x^{\top}\left(D(G)-D\left(G^{\prime}\right)\right) x \\
& \geq \sum_{i=1}^{2} \sigma_{G^{\prime}}\left(S_{i}\right)\left(\sigma_{G^{\prime}}\left(G_{1}\right)-x_{v_{i}}\right) \\
& \geq \sum_{i=1}^{2} \sigma_{G^{\prime}}\left(S_{i}\right)\left(x_{w}+x_{u}-x_{v_{i}}\right) \\
& >0
\end{aligned}
$$

and thus $\rho(G)>\rho\left(G^{\prime}\right)$.

The following is Lemma 3.4 of [3].

Corollary 3.8. Let $G$ be a connected graph with a chain $C$ of odd length $\ell$, where $\ell \geq 5$. Let $u v_{1}$ and $u v_{2}$ be two edges on the chain $C$. Suppose that $\operatorname{deg}_{G}(u) \geq 3$. If $G^{\prime}=G-\left\{v_{1} w: w \in N_{G}\left(v_{1}\right) \backslash\{u\}\right\}-\left\{v_{2} w: w \in\right.$ $\left.N_{G}\left(v_{2}\right) \backslash\{u\}\right\}+\left\{u w: w \in\left(N_{G}\left(v_{1}\right) \cup N_{G}\left(v_{2}\right)\right) \backslash\{u\}\right\}$, then $\rho(G)>\rho\left(G^{\prime}\right)$.

Proof. Let $G_{1}$ be the component of $G-E(C)$ containing $u$, and $G_{2}$ be the subgraph of $G$ induced by $V(G) \backslash$ $\left(V\left(G_{1}\right) \backslash\{u\}\right)$. Let $w \in V\left(G_{2}\right) \backslash\{u\}$. Denote by $z$ the vertex on $C$ such that its distance to $w$ is minimum among all vertices of $C$. It is evident that $z=w$ if $w$ lies on $C$. Then $d_{G}\left(v_{1}, w\right)-d_{G}\left(v_{2}, w\right)=d_{G}\left(v_{1}, z\right)-d_{G}\left(v_{2}, z\right)$. As $C$ is a chain, the shortest path connecting $v_{1}\left(v_{2}\right.$, respectively) and $z$ contains only vertices on $C$. Let $P(Q$, respectively) be the shortest path connecting $v_{1}\left(v_{2}\right.$, respectively) and $z$ in $G$. If $P$ and $Q$ are edge disjoint, then $d_{G}\left(v_{1}, z\right)+d_{G}\left(v_{2}, z\right)=\ell-2$, and as $\ell$ is odd, we have $d_{G}\left(v_{1}, z\right) \neq d_{G}\left(v_{2}, z\right)$. Otherwise, $\left|d_{G}\left(v_{1}, z\right)-d_{G}\left(v_{2}, z\right)\right|=$ $d_{G}\left(v_{1}, v_{2}\right)=2$, so $d_{G}\left(v_{1}, z\right) \neq d_{G}\left(v_{2}, z\right)$. In either case, $d_{G}\left(v_{1}, w\right) \neq d_{G}\left(v_{2}, w\right)$. So the result follows from Theorem 3.7.

Corollary 3.9. Let $H$ be a graph consisting of two nontrivial connected graphs $H_{1}$ and $H_{2}$ sharing a unique vertex $u$ such that $E(H)=E\left(H_{1}\right) \cup E\left(H_{2}\right)$. Suppose that $u v_{1}, \ldots, u v_{k}$ are pendant edges in $H_{1}$, where $k \geq 2$ and that $N_{H_{2}}(u)=N_{1} \cup N_{2}$, where $N_{1}, N_{2} \neq \emptyset$ and $N_{1} \cap N_{2}=\emptyset$. Let

$$
G=H-\left\{u w: w \in N_{H_{2}}(u)\right\}+\left\{v_{k-1} w: w \in N_{1}\right\}+\left\{v_{k} w: w \in N_{2}\right\} .
$$

For any vertex $w \in V\left(H_{2}\right) \backslash\{u\}$, if all the paths from $u$ to $w$ with length $d_{G}(u, w)$ pass only through $N_{1}$ or pass only through $N_{2}$, then $\rho(G)>\rho(H)$.

Proof. Let $G_{1}=H_{1}-\left\{v_{k-1}, v_{k}\right\}$ and let $G_{2}$ be the subgraph of $G$ induced by $V\left(H_{2}\right) \cup\left\{v_{k-1}, v_{k}\right\}$. Suppose that $z \in V\left(G_{2}\right) \backslash\{u\}$, i.e., $z \in\left(V\left(H_{2}\right) \backslash\{u\}\right) \cup\left\{v_{k-1}, v_{k}\right\}$. Note that any shortest path from $u$ to $z$ in $H_{2}$ goes through only vertices in $N_{1}$ or $N_{2}$. Correspondingly, any shortest path from $u$ to $z$ in $G_{2}$ goes through only vertices in $N_{1}$ or $N_{2}$. Consequently, $d_{G}\left(v_{k-1}, z\right) \neq d_{G}\left(v_{k}, z\right)$. Now by Theorem 3.7, $\rho(G)>\rho(H)$.

We remark that Corollary 3.9 and Theorem 3.7 are equivalent. If $k \geq 3$, then Corollary 3.9 is just Theorem 2.3 of $[16]$. 


\section{GRAphs minimizing the DistanCE SPECTRAL RADIUS}

First we determine the graphs that minimize the distance spectral radius among all homeomorphically irreducible unicylic graphs on $n \geq 6$ vertices.

Lemma 4.1 ([9]). For $k \geq 2$ and $1 \leq a_{1} \leq a_{2}-2$, let $G$ be a graph obtained from a connected graph $G_{0}$ with two vertices $u_{1}$ and $u_{2}$ such that $N_{G_{0}}\left(u_{1}\right) \backslash\left\{u_{2}\right\} \subseteq N_{G_{0}}\left(u_{2}\right) \backslash\left\{u_{1}\right\}$, by attaching $a_{i}$ pendant vertices to $u_{i}$ for each $i=1,2$. Let $G^{\prime}$ be the graph obtained from $G$ by moving one pendant edge at $u_{2}$ from $u_{2}$ to $u_{1}$. Then $\rho(G)<\rho\left(G^{\prime}\right)$.

Let $U_{n}$ be a unicylic graph on $n$ vertices obtained from a triangle $K_{3}$ with $V\left(K_{3}\right)=\left\{v_{1}, v_{2}, v_{3}\right\}$, by attaching a pendant vertex to $v_{i}$ for $i=1,2$, respectively, and attaching $n-5$ pendant vertices to $v_{3}$.

Theorem 4.2. Let $G$ be a homeomorphically irreducible unicylic graph on $n \geq 6$ vertices. Then $\rho(G) \geq \rho\left(U_{n}\right)$ with equality if and only if $G \cong U_{n}$.

Proof. Let $G$ be a homeomorphically irreducible unicylic graph on $n$ vertices that minimizes the distance spectral radius.

Let $g$ be the girth of the unique cycle $C$ of $G$. Let $u$ be a vertex on $C$. Since $G$ is a homeomorphically irreducible unicylic graph, we have $\operatorname{deg}_{G}(u) \geq 3$. Let $v_{1}, v_{2}$ be two neighbors of $u$ on $C$.

Suppose that $g \geq 4$. Suppose that $g$ is even. Let $G^{\prime}$ be the graph obtained from $G$ by moving all the edges at $v_{1}$ except $u v_{1}$ from $v_{1}$ to $u$. Note that $G^{\prime}$ is a homeomorphically irreducible unicylic graph on $n$ vertices. By Theorem 3.2 or Corollary 3.4, $\rho\left(G^{\prime}\right)<\rho(G)$, a contradiction. Thus $g$ is odd. Let $G^{\prime \prime}$ be the graph obtained from $G$ by moving all the edges at $v_{i}$ except $u v_{i}$ from $v_{i}$ to $u$ for each $i=1,2$. Obviously, $G^{\prime \prime}$ is a homeomorphically irreducible unicylic graph on $n$ vertices. By Theorem 3.7 or Corollary 3.8, we have $\rho\left(G^{\prime \prime}\right)<\rho(G)$, also a contradiction. It thus follows that $g=3$.

Suppose that $G$ has an edge, say $v w$, outside $C$ that is not a pendant edge. Evidently, $v w$ is a cut edge of $G$. Let $G^{*}$ be the graph obtained from $G$ by moving all the edges at $w$ except $v w$ from $w$ to $v$. It is obvious that $G^{*}$ is a homeomorphically irreducible unicylic graph on $n$ vertices. By Theorem 3.2 or Corollary $3.3, \rho\left(G^{*}\right)<\rho(G)$, a contradiction. Thus, every edge of $G$ outside $C$ is a pendant edge. That is, $G$ is a unicylic graph obtainable from a triangle $K_{3}$ with $V\left(K_{3}\right)=\left\{v_{1}, v_{2}, v_{3}\right\}$ by attaching $a_{i}$ pendant vertices to $v_{i}$ for $i=1,2,3$, where $1 \leq a_{1} \leq a_{2} \leq a_{3}$.

If $n=6,7$, then $G \cong U_{n}$.

Suppose that $n \geq 8$ and $a_{2} \geq 2$. Let $\widetilde{G}$ be the graph obtained from $G$ by moving one pendant edge at $v_{2}$ from $v_{2}$ to $v_{3}$. Obviously, $\widetilde{G}$ is a homeomorphically irreducible unicylic graph on $n$ vertices. By Lemma 4.1, $\rho(\widetilde{G})<\rho(G)$, a contradiction. So $a_{2}=1$. That is, $a_{1}=a_{2}=1$ and $a_{3}=n-5$, i.e., $G \cong U_{n}$.

In the following, we determine the trees that minimize the distance spectral radius among all trees on $n$ vertices with given number of vertices of degree two.

Let $G$ be a connected graph with $v \in V(G)$. For $k, \ell \geq 0$, let $G(v, k, \ell)$ be the graph obtained from $G$ by attaching two paths $P_{k}$ and $P_{\ell}$ at one end vertices to $v$. The following lemma was established in [13], for which a simple argument was given in [15].

Lemma 4.3. Let $G$ be a connected graph with $v \in V(G)$. If $k \geq \ell \geq 1$, then $\rho(G(v, k, \ell))<\rho(G(v, k+1, \ell-1))$.

A tree is called starlike if it has exactly one vertex of degree at least three; this vertex is called the branching vertex. If the branch vertex has degree $s$, we call it an $s$-starlike tree. For an $s$-starlike tree $T$ on $n$ vertices with branching vertex $u$, each path connecting $u$ and a pendant vertex is called a leg. Denote by $a_{1}, \ldots, a_{s}$ the lengths of the $s$ legs of $T$. Then $a_{1}+\cdots+a_{s}=|E(T)|=n-1$. Assume that $a_{1} \geq \cdots \geq a_{s}$. If $a_{1}-a_{s}=0,1$, then the multiset $\left\{a_{1}, \ldots, a_{s}\right\}$ composes of $\left\lfloor\frac{n-1}{s}\right\rfloor+1$ with multiplicity $r$ and $\left\lfloor\frac{n-1}{s}\right\rfloor$ with multiplicity $s-r$, where $r=n-1-s\left\lfloor\frac{n-1}{s}\right\rfloor$. In this case, we call it an $s$-starlike tree of almost equal leg lengths, denoted by $S_{n, s}$. 
Let $T$ be a tree on $n$ vertices with $t$ vertices of degree two. If $t=n-2$, then $T \cong P_{n}$. Note that $t=$ $n-3$ is impossible, since $T$ has at least two pendant vertices, and the remaining unique vertex has degree $2(n-1)-2(n-3)-1 \cdot 2=2$.

Theorem 4.4. Let $T$ be a tree on $n$ vertices with $t$ vertices of degree two, where $0 \leq t \leq n-4$. Then $\rho(T) \geq$ $\rho\left(S_{n, n-t-1}\right)$ with equality if and only if $T \cong S_{n, n-t-1}$.

Proof. Let $T$ be a tree on $n$ vertices with $t$ vertices of degree two that minimizes the distance spectral radius. Since $0 \leq t \leq n-4$, the maximum degree of $T$ is at least three.

Suppose that there are at least two vertices of degree at least three in $T$. Then we choose two such vertices, say $u$ and $v$, by requiring that the distance between them is as small as possible. Let $P$ be the path connecting $u$ and $v$. If $u$ and $v$ are not adjacent, then each vertex on $P$ except $u$ and $v$ has degree two. Let $w$ be the vertex adjacent to $v$ on $P\left(w=u\right.$ if $u$ and $v$ are adjacent). Let $T^{\prime}$ be the tree obtained from $T$ by moving all the edges at $v$ except $w v$ from $v$ to $u$. It is easily seen that $T^{\prime}$ possesses $t$ vertices of degree two. By Theorem 3.6, $\rho\left(T^{\prime}\right)<\rho(T)$, a contradiction. Thus, $T$ has exactly one vertex of degree at least three. That is, $T$ is an $s$-starlike tree for some $s$. Assume $a_{1}, \ldots, a_{s}$ are the lengths of the legs, where $a_{1} \geq \cdots \geq a_{s}$. Then $\sum_{i=1}^{s} a_{i}=n-1$ and $\sum_{i=1}^{s}\left(a_{i}-1\right)=t$. So $s=n-t-1$. By Lemma $4.3, a_{1}-a_{n-t-1}=0,1$. That is, $T$ is an $(n-t-1)$-starlike tree of almost equal leg lengths, or $T \cong S_{n, n-t-1}$.

Acknowledgements. We thank the referees for careful reading and constructive comments on the manuscript. This work was supported by the Youth Innovative Talent Project of Guangdong Province of China (No. 2020KQNCX160) and the Basic and Applied Basic Research Project of Guangzhou Municipality of China (No. 202102080685).

\section{REFERENCES}

[1] M. Aouchiche and P. Hansen, Distance spectra of graphs: a survey. Linear Algebra App. 458 (2014) 301-386.

[2] S.S. Bose, M. Nath and S. Paul, Distance spectral radius of graphs with $r$ pendent vertices. Linear Algebra App. 435 (2011) 2828-2836.

[3] S.S. Bose, M. Nath and S. Paul, On the distance spectral radius of cacti. Linear Algebra App. 437 (2012) $2128-2141$.

[4] Y. Deng, D. Li, H. Lin and B. Zhou, Distance spectral radius of series-reduced trees with parameters. RAIRO: OR 55 (2021) S2561-S2574.

[5] R.L. Graham and H.O. Pollack, On the addressing problem for loop switching. Bell Syst. Tech. J. 50 (1971) $2495-2519$.

[6] F. Harary and G. Prins, The number of homeomorphically irreducible trees, and other spices. Acta Math. 101 (1959) 141-162.

[7] A. Ilić, Distance spetral radius of trees with given matching number. Discrete Appl. Math. 158 (2010) $1799-1806$.

[8] H. Lin and B. Zhou, Distance spectral radius of trees with given number of segments. Linear Algebra App. 600 (2020) 40-59.

[9] W. Lin, Y. Zhang, Q. Chen, J. Chen, C. Ma and J. Chen, Ordering trees by their distance spectral radii. Discrete Appl. Math. 203 (2016) 106-110.

[10] H. Minc, Nonnegative Matrices. John Wiley \& Sons, New York (1988).

[11] M. Nath and S. Paul, On the distance spectral radius of bipartite graphs. Linear Algebra App. 436 (2012) $1285-1296$.

[12] S.N. Ruzieh and D.L. Powers, The distance spectrum of the path $P_{n}$ and the first distance eigenvector of connected graphs. Linear Multilinear Algebra 28 (1990) 75-81.

[13] D. Stevanović and A. Ilić, Distance spectral radius of trees with fixed maximum degree. Electron. J. Linear Algebra 20 (2010) $168-179$.

[14] Y. Wang and B. Zhou, On distance spectral radius of graphs. Linear Algebra App. 438 (2013) 3490-3503.

[15] R. Xing, B. Zhou and F. Dong, The effect of a graft transformation on distance spectral radius. Linear Algebra App. 457 (2014) 261-275.

[16] G. Yu, Y. Wu, Y. Zhang and J. Shu, Some graft transformations and its application on a distance spectrum. Discrete Math. 311 (2011) 2117-2123.

[17] B. Zelinka, Medians and peripherians of trees. Arch. Math. (Brno) 4 (1968) 87-95. 